\title{
Policy changes in Reaction Kinetics, Mechanisms and Catalysis
}

\section{Gábor Lente ${ }^{1}$}

Accepted: 14 September 2020 / Published online: 17 September 2020

(c) Akadémiai Kiadó, Budapest, Hungary 2020

This past summer brought significant changes in the general policies of our international publisher, Springer Nature. In this editorial, I wish to inform the readers of those that are directly relevant to Reaction Kinetics, Mechanisms and Catalysis.

Springer Nature has become a signatory to the Declaration on Research Assessment (DORA) [1], which recognizes the need to improve the ways in which the outputs of scholarly research are evaluated. Since the initiation of this declaration in 2012 during the Annual Meeting of the American Society for Cell Biology in San Francisco, it has become a worldwide initiative covering all scholarly disciplines and all key stakeholders including funders, publishers, professional societies, institutions, and researchers. Several funding institutions now ask the universities and other research institutions which employ the researchers they fund to embrace the principles of DORA. From now on, Springer Nature as a publisher will provide all necessary metrics and support to ensure that this journal is DORA-compliant. The editors will adhere to the DORA principles by making sure to not equate or assess research quality based on individual journal metrics.

There is a major shift in policies toward preprints, which are defined as an author's version of a research manuscript prior to formal peer review at a journal, and deposited on a public server. The widespread use of preprints, long established in some disciplines, have become an important way for researchers to share their works prior to or while under formal peer review at a journal. Preprints offer many benefits for the research community from providing free and rapid access to research findings to allowing researchers to claim priority for discovery, show evidence of progress for research assessment purposes, get community input on their work, and reap the benefits of early access to their research including accrual of citations and increased visibility. As a Springer Nature journal, we will be encouraging preprint sharing in the future. The following are the key aspects:

Gábor Lente

reac@gamma.ttk.pte.hu

1 Editorial Office, Reaction Kinetics, Mechanisms and Catalysis, Department of General and Physical Chemistry, University of Pécs, Pécs, Hungary 
- Posting a preprint of primary research will not jeopardize consideration at Reaction Kinetics, Mechanisms and Catalysis.

- Authors are free to use any license of their choosing including CC-BY licenses.

- We support citation of preprints in the reference lists. These citations should follow scholarly norms of providing credit, attribution and appropriate referencing of the literature.

- Authors are free to respond to media queries about their preprint and provide context and clarification, but we ask them to stress that the study is not peer reviewed, and findings are provisional and may change.

Further information about policy on preprint sharing, citation and licensing is available on the web [2,3].

Also, a new Springer editorial policy page has been set up to communicate to authors more efficiently [4]. This page contains links to editorial policies including authorship responsibilities and ethics, availability of data and materials.

Springer Nature is set to extend the use of Data Availability Statements to all of its journals. This is a brief, but concise, written declaration included in the published manuscript pertaining to the availability of the data generated or analyzed in the work in question. Many funding agencies require data sharing as a condition of grants, complying with these policies will be made easier by this new service.

ORCID (Open Researcher and Contributor Identifiers) is a non-profit organization that provides researchers with a unique digital identifier [5]. These identifiers can be used by editors, funding agencies, publishers, and institutions to identify individuals reliably in the same way that ISBNs and DOIs identify books and articles. Thus in a world where there are many individuals with the same first and last names, this identifier can distinguish each researcher by their unique numerical identifier. This is an invaluable asset in scientific publishing where many authors may share names, but never ORCID numbers. Springer Nature was one of the founding members of ORCID and, since 2012, has encouraged authors to submit verified ORCID identifiers on submission. In the near future, a new functionality in our submission system Editorial manager will allow ORCID sign-on at submission.

The past few months have seen a number of announcements regarding open access (OA). Most notable was that from cOAlition S, a consortium of national research agencies and funders which requires scientists and researchers funded by them to publish their work open access, confirming that 'Transformative Journals' will be an additional route to compliance for their authors. Springer Nature supports the transition to OA [6] because of the benefits it provides to authors, readers and the wider drive to open science, but I am aware that it is an uncertain development for many partners. Here I wish to confirm the fact that Reaction Kinetics and Mechanism will maintain its current status as an Open Publish journal, where payment of a publishing fee from the author is only required when the Open Access option is chosen after the acceptance of a manuscript. However, Springer Nature has an impressive set of pioneering Transformative (Read and Publish) Deals, which provides the researchers of participating institutions with OA publication rights in exchange for institutional subscriptions, without extra cost to the authors. Check out 
the details: your employer may very well be among those who already have these deals with the Publisher.

\section{References}

1. https://sfdora.org/

2. https://www.springernature.com/gp/policies/editorial-policies

3. https://www.springernature.com/gp/advancing-discovery/blog/blogposts/maximising-the-benefitsof-early-sharing/16744616

4. https://www.springer.com/gp/editorial-policies

5. https://orcid.org/

6. https://www.springernature.com/gp/open-research/policies/journal-policies

Publisher's Note Springer Nature remains neutral with regard to jurisdictional claims in published maps and institutional affiliations. 